\title{
Zebrafish erythropoiesis and the utility of fish as models of anemia
}

\author{
Kasem Kulkeaw and Daisuke Sugiyama*
}

\begin{abstract}
Erythrocytes contain oxygen-carrying hemoglobin to all body cells. Impairments in the generation of erythrocytes, a process known as erythropoiesis, or in hemoglobin synthesis alter cell function because of decreased oxygen supply and lead to anemic diseases. Thus, understanding how erythropoiesis is regulated during embryogenesis and adulthood is important to develop novel therapies for anemia. The zebrafish, Danio rerio, provides a powerful model for such study. Their small size and the ability to generate a large number of embryos enable largescale analysis, and their transparency facilitates the visualization of erythroid cell migration. Importantly, the high conservation of hematopoietic genes among vertebrates and the ability to successfully transplant hematopoietic cells into fish have enabled the establishment of models of human anemic diseases in fish. In this review, we summarize the current progress in our understanding of erythropoiesis on the basis of zebrafish studies and highlight fish models of human anemias. These analyses could enable the discovery of novel drugs as future therapies.
\end{abstract}

\section{Introduction}

Red blood cells, or erythrocytes, carry hemoglobin to supply oxygen to all tissues and organs. Approximately $2 \times 10^{13}$ erythrocytes circulate throughout the whole body. In humans, more than $10^{11}$ new erythrocytes are generated daily from bone marrow (BM) through a process known as erythropoiesis [1]. In the $\mathrm{BM}$, the hierarchy of erythropoiesis is topped by hematopoietic stem cells (HSCs), which first differentiate into common

\footnotetext{
*Correspondence: ds-mons@yb3.so-net.ne.jp

Division of Hematopoietic Stem Cells, Advanced Medical Initiatives, Department of Advanced, Medical Initiatives, Faculty of Medical Sciences, Kyushu University,

Station for Collaborative Research 1, 4F, 3-1-1 Maidashi, Higashi-Ku,

Fukuoka 812-8582 Japan
}

myeloid and common lymphoid progenitors. Common lymphoid and myeloid progenitors give rise to the adaptive and innate immune systems, respectively. Common myeloid progenitors differentiate into megakaryocyte/ erythroid progenitors and granulocyte/monocyte progenitors. At the same time, common lymphoid progenitors differentiate into B lymphocytes, $\mathrm{T}$ lymphocytes, and natural killer cells. Megakaryocyte/erythroid progenitors later give rise to erythrocytes or thrombocytes (platelets), whereas granulocyte/monocyte progenitors give rise to granulocytes (neutrophils, eosinophils, and basophils), monocytes, and dendritic cells (Figure 1). Erythrocytes synthesize hemoglobin, which is composed of two $\beta$ globin subunits and two $\alpha$-globin subunits that interact with an iron-containing heme moiety. Intrinsic transcription factors and extrinsic signaling molecules coordinately regulate erythroid differentiation and hemoglobin synthesis. Impaired erythrocyte production or hemoglobin synthesis results in anemia and decreases the oxygen supply throughout the body, a condition known as hypoxia. Much of what we know about human erythropoiesis and anemic diseases comes from studies using animal models such as Xenopus, zebrafish, chicks, and mice [2-5].

Zebrafish (Danio rerio) is a teleost freshwater fish widely distributed throughout tropical and subtropical areas of South Asia, including India, Nepal, Bangladesh, and Northern Burma [6]. Zebrafish are known worldwide as models for the study of development, cell biology, physiology, and genetics. In vivo analyses using zebrafish have some advantages over those using mice. First and foremost, zebrafish produce large numbers of small-sized embryos, permitting drug screening and functional analysis of specific genes on a large scale. Second, zebrafish have a short life span (42 to 66 months) [7] and develop rapidly, requiring 90 days to develop into adults [7], shortening periods required for experiments. Third, zebrafish embryos are transparent and develop outside a uterus, enabling researchers to view zebrafish development and genetically manipulate embryos under a microscope. Finally, many zebrafish gene functions are conserved in mice and humans, enabling researchers to translate results obtained in zebrafish studies to mammalian 


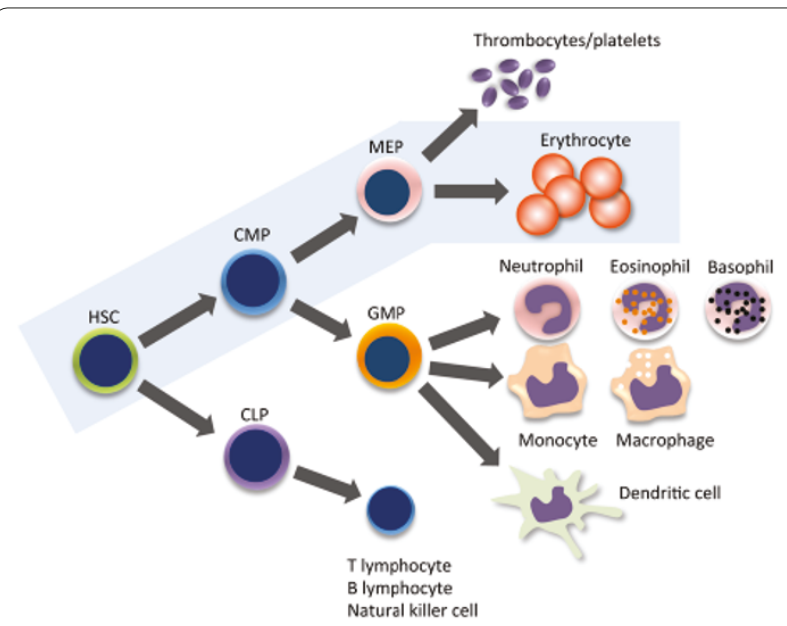

Figure 1. Schematic representation of hematopoiesis in mouse and human bone marrow. Erythropoiesis is shown in the blue box. CLP, common lymphoid progenitor; CMP, common myeloid progenitor; GMP, granulocyte-macrophage progenitor; HSC, hematopoietic stem cell; MEP, megakaryocyte-erythroid progenitor.

contexts. Currently, through large-scale mutagenesis, several models of human anemic diseases have been established in zebrafish, enabling us to develop novel therapies in anemias.

\section{Development of zebrafish erythropoiesis}

Like the generation of other blood cell types, zebrafish erythropoiesis takes place in the mesodermal germ layer and is classified into two sequential waves: primitive and definitive. The primitive wave generates erythrocytes and macrophages during embryonic development, whereas the definitive wave produces definitive HSCs, which can differentiate into every blood cell type (namely, erythrocytes, granulocytes, lymphocytes, and platelets), and maintains homeostasis throughout the zebrafish lifetime (Figure 2). To understand how erythropoiesis develops embryonically and is maintained in the adult, we also discuss the origin of erythrocytes from HSCs.

\subsection{Primitive erythropoiesis}

The fertilized egg, or zygote, divides and forms three germ layers: ectoderm, mesoderm, and endoderm; this process is known as gastrulation. Mesoderm gives rise to muscle, notochord, hematopoietic cells, pronephros, and blood vessels. Mesoderm is divided into dorsal and ventral mesoderm. Dorsal mesoderm develops into the notochord, whereas ventral mesoderm gives rise to hematopoietic cells, the pronephros, and blood vessels. Primitive hematopoiesis intraembryonically starts in ventral mesoderm-derived tissue, known as the intermediate cell mass (ICM) (Figures 2 and 3). The ICM is located between the somites and yolk sac and consists of anterior and posterior ICM (Figure 3). At the two-somite stage, which is equivalent to 10 to 11 hours post fertilization (hpf), genes encoding transcription factors required for hematopoietic cell specification, such as T-cell acute lymphocytic leukemia 1 (tal1), GATAbinding protein 2a (gata2a), and LIM domain only 2 (lmo2), and vasculogenesis, such as ets variant gene 2 $($ etv2), are co-expressed in both the anterior and posterior ICM, implying the existence of a common ancestor of hematopoietic and endothelial cells, known as the hemangioblast [8-10] (Figure 4). These genes are highly conserved among vertebrates [10-13]. Among proteins encoded by these genes, tal1, previously known as stem cell leukemia (scl), is a basic helix-loop-helix transcription factor required for both primitive and definitive hematopoiesis as well as endothelial cell differentiation, whereas gata2a is a zinc finger transcription factor functioning in proliferation and maintenance of hematopoietic progenitor cells (HPCs). Zebrafish $1 \mathrm{mo} 2$ is an LIM domain transcription factor that interacts with tal1 and gata2a, forming a DNAbinding complex, which activates the transcription of both hematopoietic and endothelial genes [10]. Zebrafish etv2, previously known as ets1-related protein (etsrp), is an E-twenty six (ets) domain-containing factor that activates transcription of endothelial-specific genes essential for vasculogenesis [11]. The posterior ICM expresses GATA-binding protein 1a (gata1a), which encodes an erythroid-specific transcription factor, and spleen focus-forming virus (SFFV) proviral integration oncogene spi1 (spi1), which encodes a myeloid-specific transcription factor, whereas the anterior ICM expresses only spi1 $[12,13]$. These studies suggest that the ICM has been committed to erythroid and myeloid lineage. Between 12 and $24 \mathrm{hpf}$, the anterior ICM develops into myeloid cells (macrophages), whereas the posterior ICM develops primarily into erythroid and some myeloid cells (Figure 3).

At 20 to 24 hpf, primitive erythroid cells (proerythroblasts and erythroblasts) expressing gata1a are present in the posterior ICM [14] (Figure 2). Similar to the mammalian yolk sac, primitive erythroid cells are surrounded by endothelial cells [15]. After the onset of blood circulation at $24 \mathrm{hpf}$, gatala ${ }^{+}$primitive erythroid cells migrate throughout the embryo and differentiate into mature erythrocytes expressing aminolevulinate, delta-, synthetase 2 (alas 2 ), which is an enzyme required for heme synthesis, and embryonic globin genes [16]. Unlike similar cells in humans and mice, zebrafish erythrocytes are nucleated and oval in shape (Figure 4). Primitive erythropoiesis accounts for all circulating erythrocytes for the first 4 days after fertilization [15].

In zebrafish, primitive erythropoiesis is regulated intrinsically by transcription factors expressed in erythroid cells and extrinsically by erythropoietin secreted from 


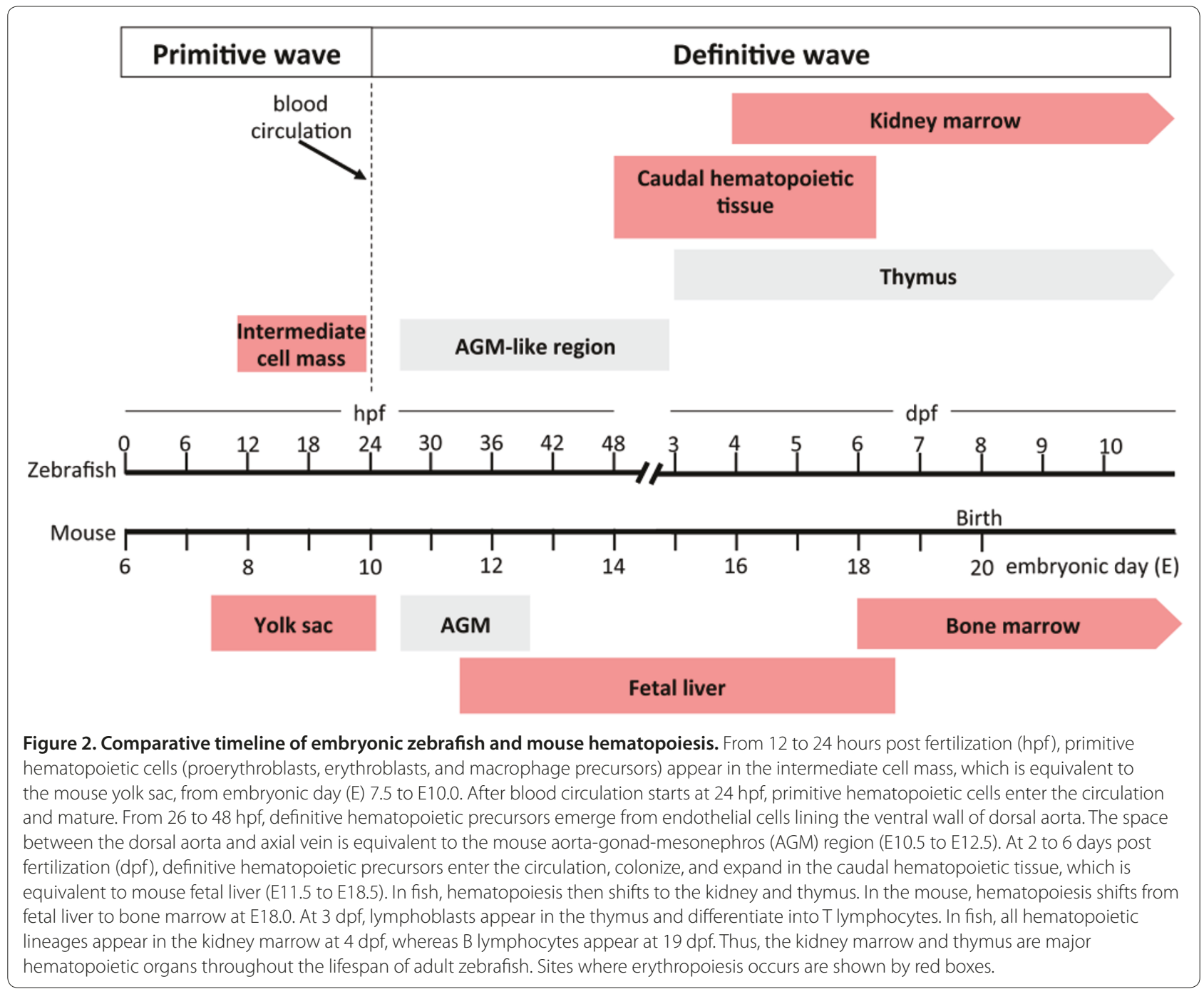

surrounding cells and tissues. Zebrafish gata1a, a zinc finger transcription factor, activates the expression of erythroid-specific genes functioning in hemoglobin synthesis, iron utilization, and cell membrane stabilization. Gata1a also suppresses the expression of myeloidspecific genes [14]. As in the mouse, a nonsense mutation in the gene encoding zebrafish gata1a results in a lack of circulating erythrocytes at $26 \mathrm{hpf}$ [17], suggesting a conservation of gatala function among vertebrates. Unlike in the mouse, zebrafish primitive erythropoiesis depends on the extrinsic factor erythropoietin (epo). Epo and erythropoietin receptor (Epor) mRNA is detected in the ICM during 16 to $24 \mathrm{hpf}$. Knockdown of Epor impairs primitive erythropoiesis in fish [18].

\subsection{Definitive erythropoiesis}

In mice, definitive HSCs are defined as having the ability to reconstitute all blood cell types in lethally irradiated adult mice. Definitive HSCs are first detected in the aorta-gonad-mesonephros (AGM) region [19] and umbilical vessels [20] at mouse embryonic day 10 or 11 . Definitive HSCs enter the blood circulation and colonize fetal liver, the first site of definitive hematopoiesis, where they expand and differentiate into erythroid and myeloid cells.

In zebrafish, an AGM-like region exists along the trunk in the space between the dorsal aorta and the underlying axial vein [21-24] (Figure 3). Cell-tracking and time-lapse imaging analyses indicate that HSCs and HPCs originate directly from the ventral wall of dorsal aorta (VDA) at 26 hpf (Figure 2) [24,25]. The morphology of endothelial cells lining the VDA changes from a long flat shape to a round shape, and the cells egress into the subaortic space and enter the blood circulation via the axial vein (Figure 3), a process called endothelial-to-hematopoietic transition [24]. As in mammals, runt-related transcription factor 1 (runx1) is critical for the emergence of hematopoietic cells from endothelial cells [26]. At $24 \mathrm{hpf}$, 


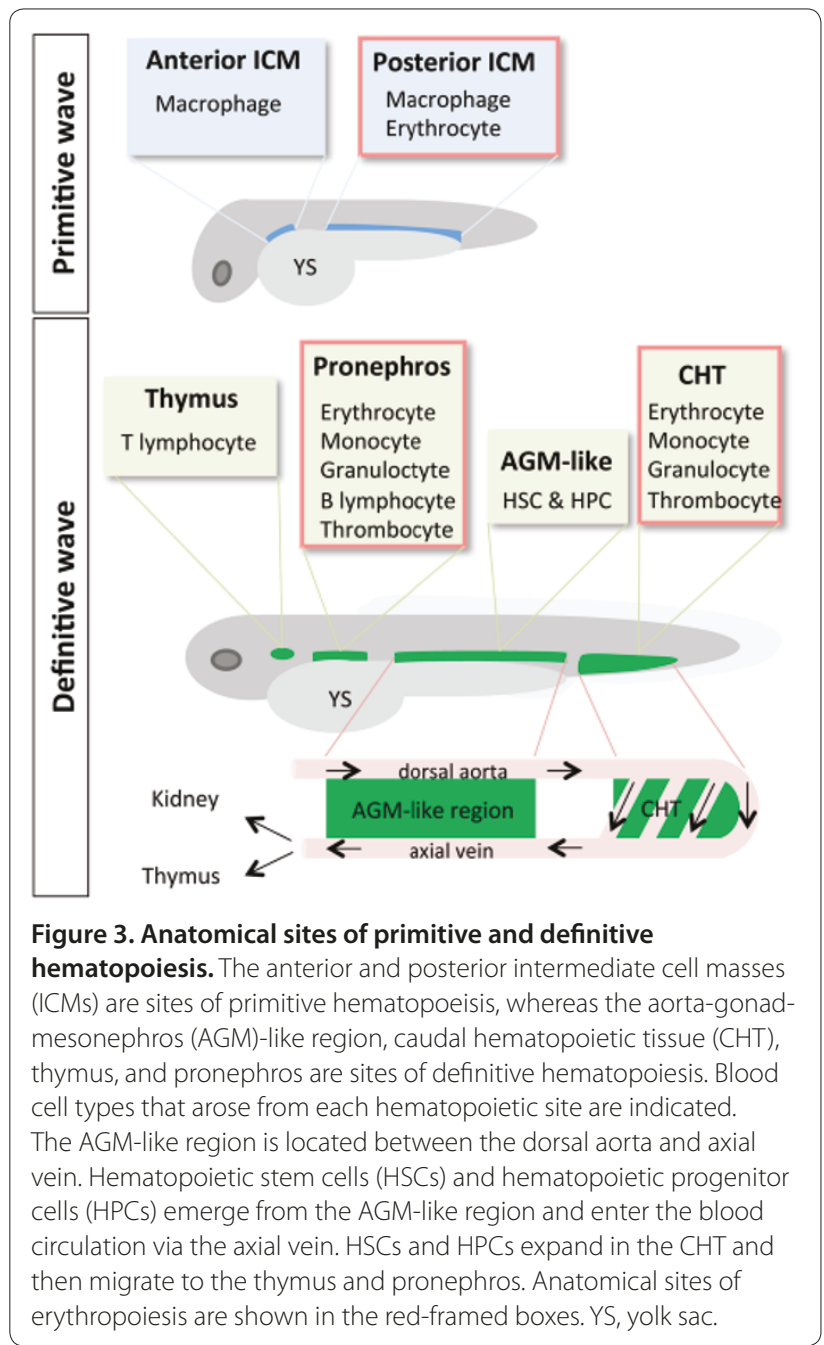

runx1-expressing HSCs and HPCs are observed in the VDA [27]. Later, at $26 \mathrm{hpf}$, dorsal aorta-derived $\operatorname{run} x 1^{+}$ HSCs and HPCs start to express the transcription factor cmyb [28], which is required for HSC migration and differentiation but not for the endothelial-to-hematopoietic transition in zebrafish [29]. Later, $c m y b^{+}$HSCs and HPCs express integrin alpha $2 \mathrm{~b}($ itga $2 b)$, also known as CD41 [30]. As in the mouse, CD41 is the earliest HSC and HPC surface marker seen in endothelial cells lining the dorsal aorta. Dorsal aorta-derived CD $41^{+} \mathrm{HSCs}$ and HPCs enter the blood circulation via the axial vein rather than the dorsal aorta and colonize caudal hematopoietic tissue by 48 hpf (Figure 3) [30]. This tissue, also known as the caudal vein plexus, is highly vascularized. From $48 \mathrm{hpf}$ to 7 days post-fertilization (dpf), $c m y b^{+} \mathrm{HSCs}$ and HPCs expand and differentiate into erythrocytes, monocyte/macrophages, and thrombocytes in caudal hematopoietic tissue (Figures 2 and 3). Therefore, caudal hematopoietic tissue exhibits properties similar to those of the mammalian fetal liver. Next, HSCs and HPCs migrate from the caudal hematopoietic tissue first to the thymus and then to the pronephros [21-23] (Figure 2). CD41 ${ }^{+}$ HSCs and HPCs first appear in the thymus at $54 \mathrm{hpf}$ (2.25 dpf) [23], and by $4 \mathrm{dpf}, c m y b^{+}$HSCs and HPCs appear in the pronephros (Figure 2), which later develops into the kidney and functions equivalently to mammalian BM $[21,22]$. Two routes of HSC migration from the AGM-like region to the pronephros have been proposed: the first to caudal hematopoietic tissue and pronephros via the circulation (Figure 3) and the second directly from the AGM-like region via the pronephric tubules [30]. At $4 \mathrm{dpf}$, only myeloerythroid lineages have progressively expanded in the kidney [15]. By $7 \mathrm{dpf}$, erythroblasts are found in the kidney [15], where they later become the major definitive hematopoietic organ of adult zebrafish.

\section{Erythropoiesis in adult zebrafish}

In mammals, adult erythropoiesis is maintained primarily in the BM. HSCs differentiate into erythroid progenitor cells and later erythroblasts. Subsequently, erythroblasts undergo terminal differentiation into mature erythrocytes. Mature erythrocytes are spherical and biconcave with a typical size of 7 to $8 \mu \mathrm{m}$. As in embryos, cellextrinsic cues, such as erythropoietin, and cell-intrinsic cues, such as erythroid-specific transcription factors Gata1 and Kruppel-like factor 1 (Klf1), coordinately regulate erythropoiesis in the BM [31].

Unlike in mammals, zebrafish erythropoiesis is maintained in the interstitium of the anterior and posterior kidney [2]. In adult kidney marrow, common myeloid progenitors differentiate into megakaryocyte/erythroid progenitors expressing the transcription factor gatala, an ortholog of mouse Gata1. The megakaryocyte/erythroid progenitors differentiate into erythroblasts. Erythroblasts later differentiate into mature erythrocytes, which are elliptical and nucleated cells with a typical size of $7 \times 10 \mu \mathrm{m}$ [32].

Although kidney marrow erythrocytes can be fractionated from blood cell mixtures by flow cytometry based on forward and side scatter (which reveal cell size and granularity, respectively), their surface markers have not been fully identified [33]. Although some potentially useful antibodies cross-react among species, it remains a challenge to analyze erythroid cells by using antibodybased techniques, owing to a shortage of reagents.

As in mammals, zebrafish erythrocytes contain hemoglobin. Human and mouse $\alpha$-globin and $\beta$-globin genes are located on separate chromosomes and arranged in order of embryonic and adult expression. The change from embryonic to adult globin expression is known as globin switching [34]. By contrast, zebrafish $\alpha$-globin and $\beta$-globin genes are located on the same chromomsome and found in embryonic and adult clusters separated by non-coding genomic DNA [35]. The embryonic cluster 


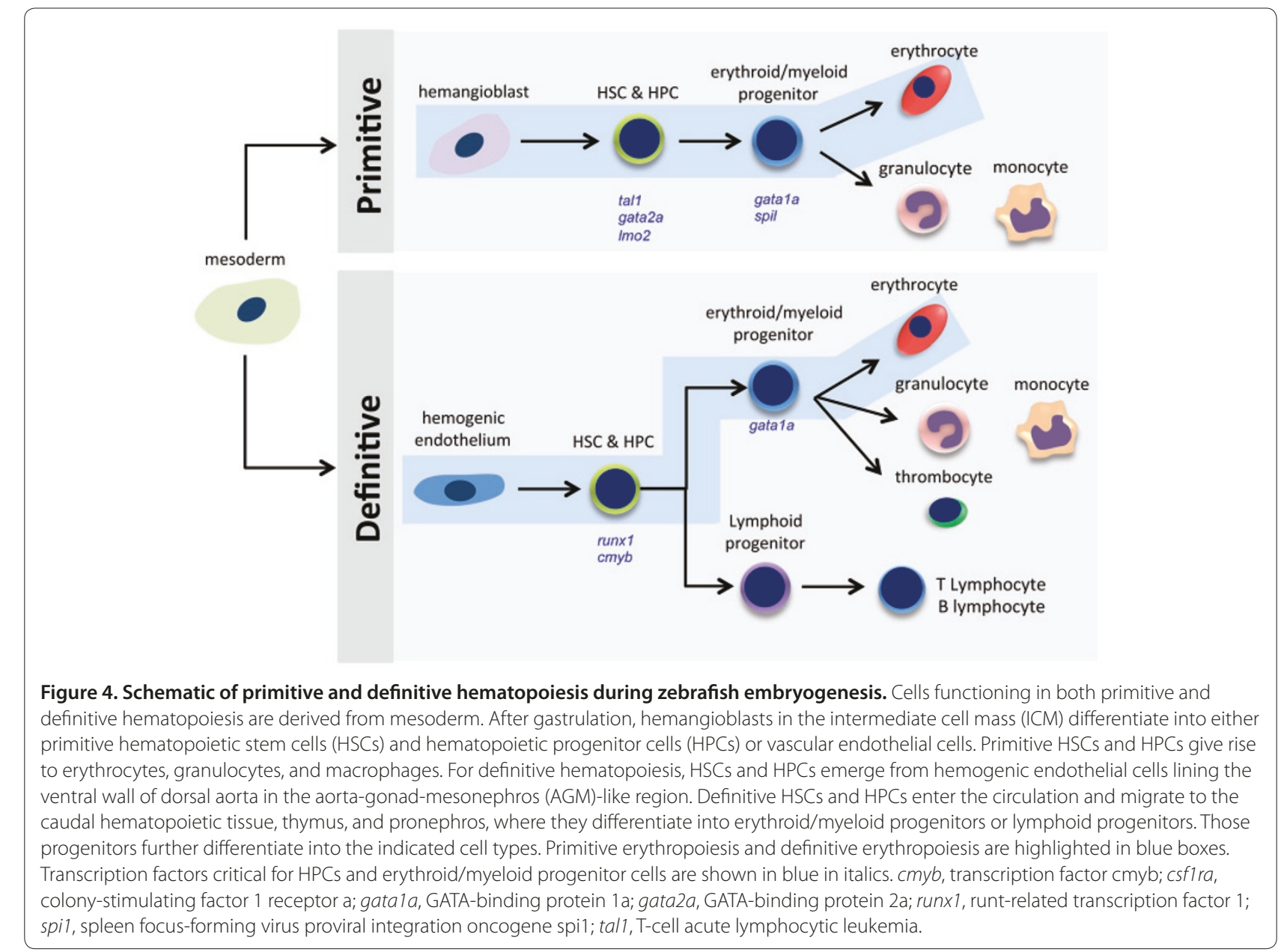

consists of hemoglobin alpha embryonic-1 (hbae1) and hemoglobin beta embryonic-1.1 (hbbe1.1), whereas the adult cluster contains hemoglobin alpha adult-1 (hbaa1) and beta adult-1 globin (ba1). Moreover, in the adult cluster, globin genes are oriented in a head-to-head pattern: $3^{\prime}-5$ ' in the case of $\alpha$-globin and 5' $-3^{\prime}$ in the case of $\beta$-globin genes. Thus, they are transcribed in the opposite direction [36]. High-performance liquid chromatography analysis shows that adult zebrafish erythrocytes in peripheral blood contain three major $\alpha$-globin and two $\beta$-globin proteins [36]. As in mammals, globin switching also occurs during zebrafish development at a stage $10 \mathrm{dpf}$ [36]. As in embryos, adult zebrafish erythropoiesis is regulated by extrinsic and intrinsic cues.

\subsection{Zebrafish erythropoietin}

Erythropoietin (Epo) is a glycoprotein crucial for survival and proliferation of erythroid progenitor cells. In mammals, there is only one Epo that is primarily produced from kidney and BM [37]. Binding of EPO to its receptor activates Janus kinase/signal transducer and activator of transcription 5 (JAK/STAT5) signaling pathway, which upregulates anti-apoptotic genes and promotes cell survival [31]. Mammalian EPO is not required for primitive erythropoiesis but is indispensable for definitive erythropoiesis $[38,39]$.

Unlike the case in mice, erythropoiesis of both primitive and definitive zebrafish depends on erythropoietin signaling [18]. Unlike the case with the mammalian Epo gene, there are three splice variants of epo gene in fish: epo-L1, epo-L2, and epo-S [40]. epo-L1 and epo- $L 2$ are expressed predominantly in the heart and liver, whereas epo-S is expressed in adult kidney marrow $[40,41]$. The C-terminal amino acid sequences of proteins encoded by these genes are identical, but the $\mathrm{N}$-terminal signal peptides differ. Misexpression studies in the monkey kidney fibroblast COS-1 cell line indicate that epo-L1 and epo-L2 are secreted but that epo-S is cytosolic [40].

In mammals, decreased blood oxygen because of anemia or hypoxia induces EPO production in the kidney and accelerates erythropoiesis. Similarly, anemia and hypoxia upregulate the expression of zebrafish epo mRNA in the heart [18]. Moreover, zebrafish erythropoietin 
Table 1. Zebrafish mutant strains relevant to human erythropoiesis-related diseases

\begin{tabular}{|c|c|c|c|c|}
\hline Human disease & Mutant & Gene & Function & Reference \\
\hline Erythropoietic protoporphyria & dracula & Ferrochelatase & Enzyme functioning in heme synthesis & {$[50]$} \\
\hline \multirow[t]{2}{*}{ Hemolytic anemia } & \multirow[t]{2}{*}{ merlot } & \multirow[t]{2}{*}{ Erythrocyte membrane protein 4.1R } & Structural protein of erythrocyte membrane & \multirow[t]{2}{*}{ [54] } \\
\hline & & & Stabilizes $\beta$-spectrin/actin interaction & \\
\hline $\begin{array}{l}\text { Congenital dyserythropoietic } \\
\text { anemia type II (HEMPAS) }\end{array}$ & retsina & $\begin{array}{l}\text { Solute carrier family } 4 \text { (anion } \\
\text { exchanger 1a or band } 3 \text { ) }\end{array}$ & Chloride exchanger & [56] \\
\hline Hereditary spherocytosis & riesling & $\beta$-spectrin & Structural protein of erythrocyte membrane & [59] \\
\hline Congenital sideroblastic anemia & sauternes & Aminolevulinate synthetase 2 (alas 2 ) & Enzyme functioning in heme synthesis & [63] \\
\hline $\begin{array}{l}\text { Hypochromic anemia } \\
\text { (resembles thalassemia) }\end{array}$ & zinfandel & Globin & Oxygen transport & {$[35]$} \\
\hline Type IV hemochromatosis & weissherbst & Ferroportin 1 & Iron transport & [65] \\
\hline
\end{tabular}

HEMPAS, hereditary erythroblastic multinuclearity with positive acidified serum lysis test.

signaling requires stat5.1 protein, an ortholog of human STAT5 [18]. These observations demonstrate that epo/ epor function is highly conserved among vertebrates.

\subsection{Zebrafish erythroid transcription factors}

In the mouse, Gata1 regulates transcription of erythropoietic genes, including Klf1. Gata1 knockout mice die during gestation because of severe anemia [42]. Like Gata1, mouse Klf1 is essential for definitive erythropoiesis in fetal liver [43]. Klf1 reportedly regulates the expression of several erythroid-specific genes encoding (a) globin, (b) enzymes for heme biosynthesis, and (c) erythroid membrane and cytoskeletal proteins [44].

Little is known about the role of gatala in adult erythropoiesis because of the lethal phenotype of null mutants at an early stage of development [17]. In addition, no functional ortholog of mouse Klf1 has been identified in zebrafish. Although the zebrafish $k l f d$ gene is expressed in both primitive and definitive erythropoietic organs [45] and the amino acid sequence of zebrafish klfd is similar to that of mouse Klf1 [45], there is currently no direct evidence that klfd functions in definitive erythropoiesis. Zebrafish klf4 knockdown using antisense morpholino oligonucleotides downregulates the expression of embryonic $\beta$-globin and genes involved in heme biosynthesis, but no defect is seen in definitive erythropoiesis [46]. Therefore, it is unlikely that klf4 is the ortholog of mouse Klf1. Thus, owing to the lack of a model, the roles of gata1a and klfd in adult erythropoiesis have not been clarified. These outcomes prompted us to establish transient and reversible downregulation of both transcription factors in adult zebrafish, as discussed in section 3.8.

\section{Zebrafish as models of human erythropoiesis-related diseases}

Zebrafish erythropoietic genes are functionally similar to those expressed in mice and humans and include genes encoding enzymes for heme biosynthesis, structural erythrocyte membrane proteins, epo/epo receptor, and globin [47]. Large-scale mutagenesis of zebrafish has enabled the identification of genes regulating hematopoiesis/erythropoiesis $[32,48]$. The human homologs of some of these genes function in hematological diseases [3]. Zebrafish are advantageous for evaluating the function of genes underlying erythropoietic disease since fish embryos are resistant to severe anemic conditions because of passive diffusion of oxygen into the fish. Fish models of human anemias (Table 1) are described below. Many of the following mutants could be useful to test new drugs.

\subsection{Erythropoietic protoporphyria}

Erythropoietic protoporphyria occurs worldwide, has a prevalence of 1:75,000 in The Netherlands [49], and is caused by ferrochelatase deficiency. Ferrochelatase catalyzes the formation of heme by transferring iron to protoporphyrin, a heme intermediate. Mutations in the human ferrochelatase gene promote protoporphyrin accumulation in the skin, erythrocytes, and liver, resulting in sensitivity to light exposed to the skin or even erythrocytes and skin burning and itching [49]. Among patients, $20 \%$ to $60 \%$ also exhibit anemia due to decreased heme synthesis and light-dependent erythrocyte lysis. Some patients ( $1 \%$ to $4 \%$ ) show liver disease due to the accumulation of free protoporphyrin released from lysed erythrocytes [49]. Although the avoidance of sun exposure and treatment with light-protective substances such as $\beta$-carotene and melanin in skin can ameliorate symptoms, no curative treatment is yet available [49].

The zebrafish mutant dracula exhibits a point mutation in the ferrochelatase gene, creating an in-frame stop codon and expression of a dysfunctional enzyme. Dracula fish manifest autofluorescent erythrocytes, light-dependent hemolysis, and liver malfunction, similar to conditions seen in humans [50]. Owing to the transparency of fish embryos and protoporphyrin autofluorescence, protoporphyrin 
accumulation can be monitored microscopically in various organs of an intact fish, an analysis impossible in humans and mice, making dracula mutants a suitable model for human erythropoietic protoporphyria.

\subsection{Hemolytic anemia}

Abnormality of erythroid cell membrane leads to massive erythrocyte destruction in the spleen, a condition known as hemolytic anemia. Human hereditary elliptocytosis is characterized by elliptical erythrocytes, in which abnormal cell membranes lead to hemolytic anemia. Human hereditary elliptocytosis occurs worldwide but is prevalent in West Africa [51]. Often patients show no symptoms, and only $10 \%$ have mild to severe anemia [51]. In human hereditary elliptocytosis, many patients harbor point mutations in the gene encoding protein 4.1R, a major component of the erythrocyte cytoskeleton that maintains biconcave morphology. These mutations promote decreased protein expression or impair protein interaction with other cytoskeletal proteins [52]. Owing to massive hemolysis, patients with hereditary elliptocytosis have complications such as cardiomegaly, splenomegaly, and gallstones. Only supportive treatments, such as folate therapy, blood transfusion, splenectomy, and gallstone removal, are currently available $[51,53]$. Although HSC transplantation is one curative therapy, novel drugs are needed to antagonize hemolysis.

The zebrafish merlot mutant exhibits severe hemolytic anemia due to mutation in the gene encoding $4.1 \mathrm{R}$ protein. Unlike mammalian erythrocytes, wild-type mature zebrafish erythrocytes exhibit both spherical and elliptical morphologies. Merlot mutants show spiculated erythrocyte membranes, resulting in hemolytic anemia and conditions such as cardiomegaly and splenomegaly, phenotypes similar to those seen in humans [54].

\subsection{Congenital dyserythropoietic anemia type II}

Human congenital dyserythropoietic anemia type II is an erythroid-specific abnormality in cell division, leading to multinuclear erythroblasts, erythroblast apoptosis (dyserythropoiesis), and anemia. The condition varies from mild to severe: approximately $15 \%$ of patients require blood transfusions during infancy and early childhood but not thereafter [55]. Splenomegaly occurs in 50\% to $60 \%$ of patients, and gallstones are frequently observed. In the $\mathrm{BM}, 10 \%$ to $45 \%$ of erythroblasts are bi- and multinucleated [55]. Dyserythropoiesis is caused by a mutation in the anion exchanger protein band 3 , which is present in the human erythrocyte membrane [55]. The zebrafish retsina mutant exhibits erythroid-specific defects in cell division because of mutation in the fish ortholog of the band 3 gene. These defects resemble those of the human disease [56]. Severely affected anemic patients require blood transfusion and HSC transplantation [57].

\subsection{Hereditary spherocytosis}

Human hereditary spherocytosis is a hemolytic anemia common in Caucasians and has a prevalence of 1:2,000 to 1:5,000 [53]. Hereditary spherocytosis is characterized by abnormal erythrocyte morphology. Normally, the shape of human erythrocytes is a biconcave disk. In hereditary spherocytosis, the erythrocytes exhibit a spherical shape, leading to their massive destruction in the spleen. Mutation in the gene encoding the cytoskeletal protein spectrin has been identified as a cause of human hereditary spherocytosis. Erythroid spectrin stabilizes membrane bilayers [58]. Anemia ranging from mild (blood transfusionindependent) to severe (blood transfusion-dependent) is the main clinical feature of this condition. Patients also exhibit hyperbilirubinemia, causing jaundice, and splenomegaly. The zebrafish riesling carries a mutant $\beta$-spectrin gene and exhibits anemia due to erythrocyte hemolysis, similar to conditions seen in humans [59]. Zebrafish $\beta$-spectrin shares $62.3 \%$ identity with the human ortholog. In addition to exhibiting abnormal cell morphology-induced hemolysis, zebrafish riesling erythrocytes undergo apoptosis, which had not been observed in human hereditary spherocytosis. Thus, analysis of the zebrafish riesling mutant has revealed a novel mechanism of erythrocyte hemolysis [59].

\subsection{Congenital sideroblastic anemia}

Human congenital sideroblastic anemia is characterized by iron deposition in mitochondria of erythroblasts in the $\mathrm{BM}$ and is caused by a mutation in the gene encoding $\delta$ aminolevulinate synthase, or ALAS2, which catalyzes the first step of heme biosynthesis. A lack of heme promotes increases in free iron levels and subsequent iron deposition in erythroblast mitochondria, causing insufficient production of mature erythrocytes [60]. Generally, patients have symptoms of anemia, such as skin paleness, fatigue, dizziness, and enlargement of the spleen and liver. In addition to a decrease of mature erythrocytes, hypochromic microcytic erythrocytes are observed in the patients. Heme reportedly promotes gene expression of $\beta$ globin through binding Batch1, a transcriptional repressor of $\beta$-globin gene $[61,62]$. Therefore, decreased intracellular heme because of mutated ALAS2 may contribute to hypochromic microcytic anemia. Mutation in the zebrafish gene sauternes, which encodes alas2 protein, results in a condition similar to hypochromic microcytic anemia in humans [63]. Sauternes mutant embryos show delayed erythrocyte maturation and decreased $\beta$-globin expression. These mutants represent the first animal model to allow the investigation of mechanisms underlying hemeinduced globin synthesis.

\subsection{Hypochromic anemia}

Hypochromic anemia is a general term for anemia in which erythrocytes look paler and smaller than they do 


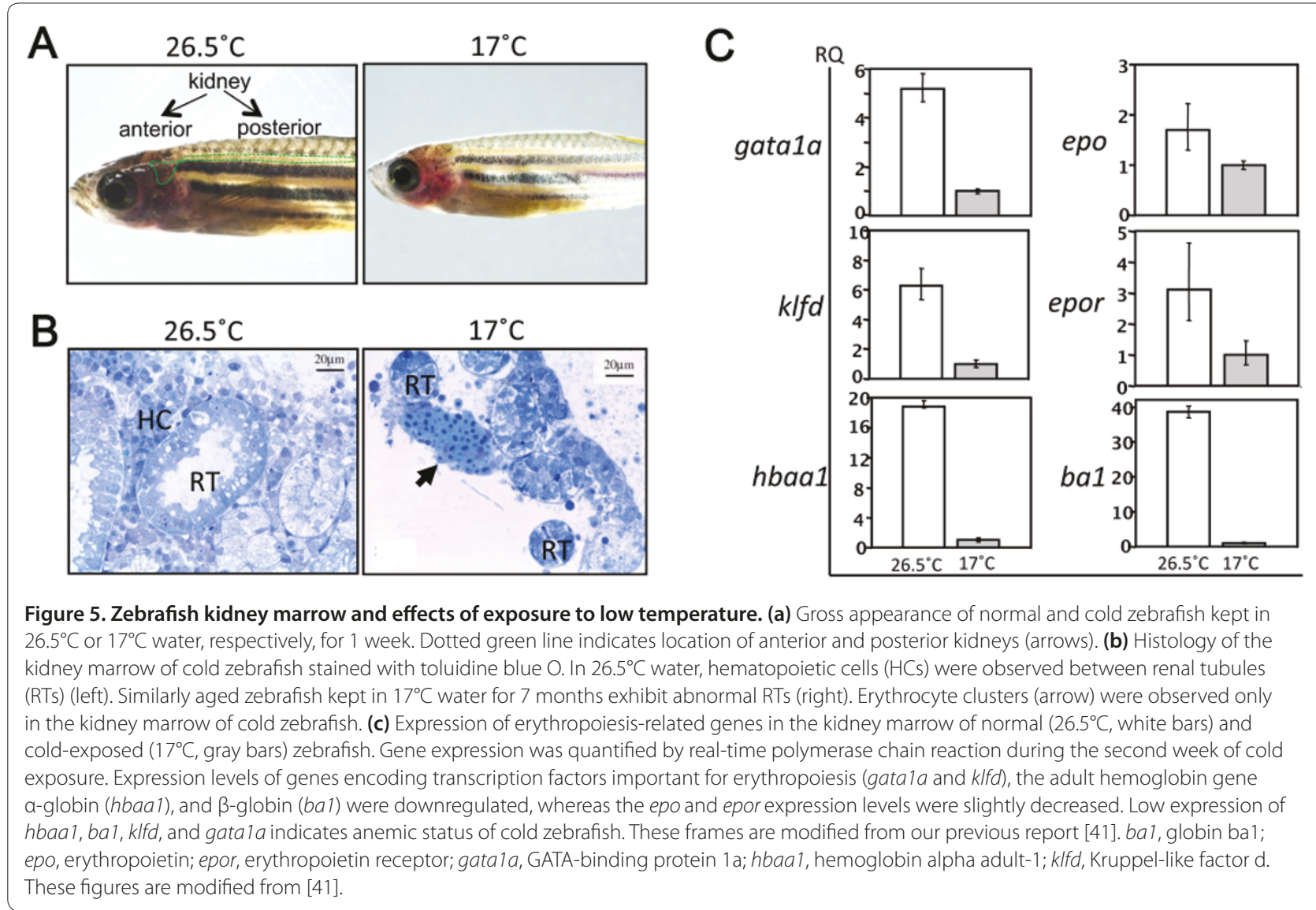

normally. In humans, hypochromic anemia results from reduction in either globin synthesis (as occurs in thalassemia) or iron absorption (as occurs in iron deficiency) or from vitamin $\mathrm{B}_{6}$ deficiency. In mammals and fish, different globin subtypes are expressed in embryonic and adult stages. In adult mammals, embryonic globin synthesis is suppressed whereas adult globin synthesis is activated [34]. Thalassemic patients show reduced adult globin synthesis and re-activate fetal globin expression, which can ameliorate anemia severity. The zebrafish zinfandel mutant exhibits hypochromic microcytic anemia due to defective embryonic globin production. This condition is rescued in adult fish once adult globin is produced [35].

\subsection{Type IV hemochromatosis (iron overload)}

Type IV hemochromatosis, or iron overload, is characterized by increased intestinal iron absorption and progressive iron deposition in various tissues, resulting in hepatic cirrhosis, arthritis, cardiomyopathy, diabetes, hypopituitarism, and/or hyperpigmentation [64]. Its primary cause is mutation in genes encoding membrane proteins functioning in iron transportation, such as hemochromatosis protein, the transferrin receptor, and hemojuvelin. Analysis of zebrafish weissherbst mutants revealed mutations in a novel iron transporter, named ferroportin 1 [65] - mutations later identified in patients with type IV hemochromatosis [66,67].

\subsection{Other anemias}

As noted, most zebrafish mutant lines with defects in erythropoiesis were generated by mutagenesis by using ethylnitrosourea $[32,48]$. These pioneering studies provided useful animal models of anemia and identified numerous genes underlying human anemias. Nonetheless, such screens take time and are expensive, prompting us to establish a novel, simpler anemic model.

It is known that temperature regulates hematopoiesis [68]. High temperature increases the number of hematopoietic cells in the BM of rats and the nine-banded armadillo (Dasypus novemcinctus), whereas low temperature has opposite effects [68]. However, in nature, zebrafish survive in a wide range of temperatures, from $6^{\circ} \mathrm{C}$ in winter to $38^{\circ} \mathrm{C}$ in summer [6], making them useful to investigate the effect of temperature on hematopoiesis. Previously, we established a novel reversible anemic model by keeping zebrafish at $17^{\circ} \mathrm{C}$ (cold zebrafish) [41]. By comparison with fish kept at a higher temperature $\left(26.5^{\circ} \mathrm{C}\right)$, 'cold' zebrafish appear paler starting at the first week of cold exposure (Figure 5a, right panel). The kidney 
marrow of cold zebrafish shows abnormally structured renal tubules and erythrocyte clusters (Figure 5b, right panel). We examined the expression of genes essential for HSC maintenance, erythropoiesis, and myelopoiesis by real-time polymerase chain reaction (PCR). Interestingly, only HSC-regulated genes (runx1, cmyb, gata2a, and tal1) (data not shown) and erythropoietic genes (gatala, klfd, epo, epor, hbaa1, and ba1) (Figure 5c) were downregulated the first week of exposure. These genes were expressed at generally lower levels by the second week, suggestive of anemia. However, expression of colonystimulating factor $1 a$, which is required for macrophage differentiation, and of colony-stimulating factor 3 (granulocyte), which is required for granulocyte differentiation, was unchanged [41]. These results suggest that cold exposure specifically suppresses erythropoiesis. Moreover, cold-induced anemia was reversed when fish were returned to $26.5^{\circ} \mathrm{C}$ conditions, implying that phenotypes are directly caused by temperature [41].

To further identify novel genes regulating erythropoiesis mediated by cold exposure, we assessed global changes in gene expression in the kidney marrow by DNA microarray (unpublished data). Although decreased temperature suppressed enzymatic activity and resulted in a global decrease in gene expression, we observed both increased and decreased gene expression in the kidney marrow of cold zebrafish, as confirmed by real-time PCR. We are now undertaking loss-of-function analysis of candidate factors by using antisense morpholino oligonucleotides and small interference RNAs in fish, and we are conducting gain-of-function analysis in mouse and human cell lines.

\section{Conclusions}

Defects in erythropoiesis result in various anemic diseases. To gain an understanding of these diseases, it is necessary to determine how erythropoiesis is regulated in normal conditions as well as to establish in vivo models. We propose that, in addition to several anemic mutants, our cold zebrafish model is a useful tool to explore novel genes functioning in erythropoiesis. Given the advantages of zebrafish models, it is feasible to reach these objectives. Knowledge of the etiology and molecular mechanisms underlying these conditions will lead to the development of novel therapies.

This article is part of a thematic series on Stem cell research in the Asia-Pacific edited by Oscar Lee, Songtao Shi, Yufang Shi, and Ying Jin. Other articles in the series can be found online at http://stemcellres.com/series/asiapacific.

\section{Abbreviations}

AGM, aorta-gonad-mesonephros; bal, globin ba1; BM, bone marrow; cmyb, transcription factor cmyb; dpf, days post-fertilization; epo, erythropoietin; Epor, erythropoietin receptor; etv2, ets variant gene 2; gata 1a, GATA-binding protein 1a; gata2a, GATA-binding protein 2a; hbaa1, hemoglobin alpha adult-1; HPC, hematopoietic progenitor cell; hpf, hours post fertilization; HSC, hematopoietic stem cell; ICM, intermediate cell mass; kIf, Kruppel-like factor; Imo2, LIM domain only 2; PCR, polymerase chain reaction; runx1, runt-related transcription factor 1 ; spi1, spleen focus-forming virus proviral integration oncogene spi1; stat5.1, signal transducer and the activator of transcription 5.1; tal1, T-cell acute lymphocytic leukemia; VDA, ventral wall of dorsal aorta.

\section{Competing interests}

The authors declare that they have no competing interests.

\section{Acknowledgments}

We thank Elise Lamar, Tohru Ishitani and Keai Sinn for editing the manuscript and acknowledge grant support from the Tokyo Biochemical Research Foundation; the Japan Society for the Promotion of Science; the Ministry of Education, Culture, Sports, Science, and Technology; and the Ministry of Health, Labor, and Welfare. KK is a recipient of a fellowship supported by the Tokyo Biochemical Research Foundation and a postdoctoral fellowship for foreign researchers by the Japan Society for the Promotion of Science.

Published: 20 December 2012

\section{References}

1. Sieff C, Williams D: Hematopoiesis. In Blood: Principles and Practice of Hematology. Edited by Handin R, Lux S, Stossel T. Philadephia: Lippincott, 1995:171-224.

2. Ciau-Uitz A, Walmsley M, Patient R: Distinct origins of adult and embryonic blood in Xenopus. Cell 2000, 102:787-796.

3. Amatruda JF, Zon LI: Dissecting hematopoiesis and disease using the zebrafish. Dev Biol 1999, 216:1-15.

4. Godin I, Cumano A: Of birds and mice: hematopoietic stem cell development. Int J Dev Biol 2005, 49:251-257.

5. Orkin SH, Zon LI: Hematopoiesis: an evolving paradigm for stem cell biology. Cell 2008, 132:631-644.

6. Spence R, Gerlach G, Lawrence, Smith C: The behavior and ecology of the zebrafish, Danio rerio. Biol Rev 2008, 83:13-34.

7. The Zebrafish Model Organism Database [http://zfin.org].

8. Stainier DY, Weinstein BM, Detrich HW 3rd, Zon LI, Fishman MC: Cloche, an early acting zebrafish gene, is required by both the endothelial and hematopoietic lineages. Development 1995, 121:3141-3150.

9. Vogeli KM, Jin SW, Martin GR, Stainier DY: A common progenitor for haematopoietic and endothelial lineages in the zebrafish gastrula. Nature 2006, 443:337-339.

10. Patterson $\sqcup$, Gering M, Eckfeldt CE, Green AR, Verfaillie CM, Ekker SC, Patient $\mathrm{R}$ : The transcription factors $\mathrm{SCl}$ and $\mathrm{Lmo} 2$ act together during development of the hemangioblast in zebrafish. Blood 2007, 109:2389-2398.

11. Gomez G, Lee JH, Veldman MB, Lu J, Xiao X, Lin S: Identification of vascular and hematopoietic genes downstream of etsrp by deep sequencing in zebrafish. PLoS One 2012, 7:e31658.

12. Detrich HW 3rd, Kieran MW, Chan FY, Barone LM, Yee K, Rundstadler JA, Pratt S, Ransom D, Zon Ll: Intraembryonic hematopoietic cell migration during vertebrate development. Proc Natl Acad Sci U S A 1995, 92:10713-10717.

13. Lieschke GJ, Oates AC, Paw BH, Thompson MA, Hall NE, Ward AC, Ho RK, Zon LI, Layton JE: Zebrafish SPI-1 (PU.1) marks a site of myeloid development independent of primitive erythropoiesis: implications for axial patterning. Dev Biol 2002, 246:274-295.

14. Galloway JL, Wingert RA, Thisse C, Thisse B, Zon LI: Loss of gata1 but not gata2 converts erythropoiesis to myelopoiesis in zebrafish embryos. Dev Cell 2005, 8:109-116.

15. Willett CE, Cortes A, Zuasti A, Zapata AG: Early hematopoiesis and developing lymphoid organs in the zebrafish. Dev Dyn 1999, 214:323-336.

16. Long Q, Meng A, Wang H, Jessen JR, Farrell MJ, Lin S: GATA-1 expression pattern can be recapitulated in living transgenic zebrafish using GFP reporter gene. Development 1997, 124:4105-4111.

17. Lyons SE, Lawson ND, Lei L, Bennett PE, Weinstein BM, Liu PP: A nonsense mutation in zebrafish gata1 causes the bloodless phenotype in vlad tepes. Proc Natl Acad Sci U S A 2002, 99:5454-5459.

18. Paffett-Lugassy N, Hsia N, Fraenkel PG, Paw B, Leshinsky I, Barut B, Bahary N, Caro J, Handin R, Zon LI: Functional conservation of erythropoietin signaling in zebrafish. Blood 2007, 110:2718-2726.

19. Mizuochi C, Fraser ST, Biasch K, Horio Y, Kikushige Y, Tani K, Akashi K, Tavian M, 
Sugiyama D: Intra-aortic clusters undergo endothelial to hematopoietic phenotypic transition during early embryogenesis. PLoS One 2012, 7:e35763.

20. Sasaki T, Mizuochi C, Horio Y, Nakao K, Akashi K, Sugiyama D. Regulation of hematopoietic cell clusters in the placental niche through SCF/Kit signaling in embryonic mouse. Development 2010, 137:3941-3952.

21. Murayama E, Kissa K, Zapata A, Mordelet E, Briolat V, Lin HF, Handin RI, Herbomel P: Tracing hematopoietic precursor migration to successive hematopoietic organs during zebrafish development. Immunity 2006, 25:963-975

22. Jin $\mathrm{H}, \mathrm{Xu} J$, Wen $\mathrm{Z}$ : Migratory path of definitive hematopoietic stem/ progenitor cells during zebrafish development. Blood 2007, 109:5208-5214.

23. Kissa K, Murayama E, Zapata A, Cortés A, Perret E, Machu C, Herbomel P: Live imaging of emerging hematopoietic stem cells and early thymus colonization. Blood 2008, 111:1147-1156.

24. Kissa K, Herbomel P: Blood stem cells emerge from aortic endothelium by a novel type of cell transition. Nature 2010, 464:112-115.

25. Bertrand JY, Chi NC, Santoso B, Teng S, Stainier DY, Traver D: Haematopoietic stem cells derive directly from aortic endothelium during development. Nature 2010, 464:108-111.

26. Kalev-Zylinska ML, Horsfield JA, Flores MV, Postlethwait JH, Vitas MR, Baas AM, Crosier PS, Crosier KE: Runx1 is required for zebrafish blood and vessel development and expression of a human RUNX1-CBF2T1 transgene advances a model for studies of leukemogenesis. Development 2002, 129:2015-2030

27. Burns CE, DeBlasio T, Zhou Y, Zhang J, Zon L, Nimer SD: Isolation and characterization of runxa and runxb, zebrafish members of the runt family of transcriptional regulators. Exp Hematol 2002, 30:1381-1389.

28. Gering M, Patient R: Hedgehog signaling is required for adult blood stem cell formation in zebrafish embryos. Dev Cell 2005, 8:389-400.

29. Zhang Y, Jin H, Li L, Qin FX, Wen Z: cMyb regulates hematopoietic stem/ progenitor cell mobilization during zebrafish hematopoiesis. Blood 2011, 118:4093-4101.

30. Bertrand JY, Kim AD, Teng S, Traver D: CD41+ cmyb+ precursors colonize the zebrafish pronephros by a novel migration route to initiate adult hematopoiesis. Development 2008, 135:1853-1862.

31. Hattangadi SM, Wong P, Zhang L, Flygare J, Lodish HF: From stem cell to red cell: regulation of erythropoiesis at multiple levels by multiple proteins, RNAs, and chromatin modifications. Blood 2011, 118:6258-6268.

32. Ransom DG, Haffter P, Odenthal J, Brownlie A, Vogelsang E, Kelsh RN, Brand M, van Eeden FJ, Furutani-Seiki M, Granato M, Hammerschmidt M, Heisenberg CP, Jiang YJ, Kane DA, Mullins MC, Nüsslein-Volhard C: Characterization of zebrafish mutants with defects in embryonic hematopoiesis. Development 1996, 123:311-319.

33. Traver D, Paw BH, Poss KD, Penberthy WT, Lin S, Zon LI: Transplantation and in vivo imaging of multilineage engraftment in zebrafish bloodless mutants. Nat Immunol 2003, 4:1238-1246.

34. Wilber A, Nienhuis AW, Persons DA: Transcriptional regulation of fetal to adult hemoglobin switching: new therapeutic opportunities. Blood 2011, 117:3945-3953.

35. Brownlie A, Hersey C, Oates AC, Paw BH, Falick AM, Witkowska HE, Flint J, Higgs D, Jessen J, Bahary N, Zhu H, Lin S, Zon L: Characterization of embryonic globin genes of the zebrafish. Dev Biol 2003, 255:48-61.

36. Chan FY, Robinson J, Brownlie A, Shivdasani RA, Donovan A, Brugnara C, Kim J, Lau BC, Witkowska HE, Zon LI: Characterization of adult alpha- and betaglobin genes in the zebrafish. Blood 1997, 89:688-700.

37. Lin FK, Suggs S, Lin CH, Browne JK, Smalling R, Egrie JC, Chen KK, Fox GM, Martin F, Stabinsky Z: Cloning and expression of the human erythropoietin gene. Proc Natl Acad Sci U S A 1985, 82:7580-7584.

38. Lin CS, Lim SK, D'Agati V, Costantini F: Differential effects of an erythropoietin receptor gene disruption on primitive and definitive erythropoiesis. Genes Dev 1996, 10:154-164.

39. Lee R, Kertesz N, Joseph SB, Jegalian A, Wu H: Erythropoietin (Epo) and EpoR expression and 2 waves of erythropoiesis. Blood 2001, 98:1408-1415.

40. Chu CY, Cheng $\mathrm{CH}$, Yang $\mathrm{CH}$, Huang $\mathrm{CJ}$ : Erythropoietins from teleosts. Cell Mol Life Sci 2008, 65:3545-3552

41. Kulkeaw K, Ishitani T, Kanemaru T, Fucharoen S, Sugiyama D: Cold exposure down-regulates zebrafish hematopoiesis. Biochem Biophys Res Commun 2010, 394:859-864.

42. Fujiwara Y, Browne CP, Cunniff K, Goff SC, Orkin SH: Arrested development of embryonic red cell precursors in mouse embryos lacking transcription factor GATA-1. Proc Natl Acad Sci U S A 1996, 93:12355-12358

43. Perkins $A C$, Sharpe $A H$, Orkin SH: Lethal beta-thalassaemia in mice lacking the erythroid CACCC-transcription factor EKLF. Nature 1995, 375:318-322.

44. Hodge D, Coghill E, Keys J, Maguire T, Hartmann B, McDowall A, Weiss M, Grimmond S, Perkins A: A global role for EKLF in definitive and primitive erythropoiesis. Blood 2006, 107:3359-3370.

45. Oates AC, Pratt SJ, Vail B, Yan YI, Ho RK, Johnson SL, Postlethwait JH, Zon LI: The zebrafish klf gene family. Blood 2001, 98:1792-1801

46. Gardiner MR, Gongora MM, Grimmond SM, Perkins AC: A global role for zebrafish klf4 in embryonic erythropoiesis. Mech Dev 2007, 124:762-774.

47. Davidson AJ, Zon LI: The 'definitive' (and 'primitive') guide to zebrafish hematopoiesis. Oncogene 2004, 23:7233-7246.

48. Weinstein BM, Schier AF, Abdelilah S, Malicki J, Solnica-Krezel L, Stemple DL, Stainier DY, Zwartkruis F, Driever W, Fishman MC: Hematopoietic mutations in the zebrafish. Development 1996, 123:303-309.

49. Lecha M, Puy H, Deybach JC: Erythropoietic protoporphyria. Orphanet J Rare Dis 2009, 4:19

50. Childs S, Weinstein BM, Mohideen MA, Donohue S, Bonkovsky H, Fishman $\mathrm{MC}$ : Zebrafish dracula encodes ferrochelatase and its mutation provides a model for erythropoietic protoporphyria. Curr Biol 2000, 10:1001-1004.

51. An X, Mohandas N: Disorders of red cell membrane. Br J Haematol 2008, 141:367-375.

52. Lorenzo F, Dalla Venezia N, Morlé L, Baklouti F, Alloisio N, Ducluzeau MT, Roda $L$, Lefrançois $P$, Delaunay J: Protein 4.1 deficiency associated with an altered binding to the spectrin-actin complex of the red cell membrane skeleton. J Clin Invest 1994, 94:1651-1656.

53. Barcellini W, Bianchi P, Fermo E, Imperiali FG, Marcello AP, Vercellati $C$, Zaninoni A, Zanella A: Hereditary red cell membrane defects: diagnostic and clinical aspects. Blood Transfus 2011, 9:274-277.

54. Shafizadeh E, Paw BH, Foott H Liao EC, Barut BA, Cope JJ, Zon LI, Lin S: Characterization of zebrafish merlot/chablis as non-mammalian vertebrate models for severe congenital anemia due to protein 4.1 deficiency. Development 2002, 129:4359-4370.

55. Denecke J, Marquardt T: Congenital dyserythropoietic anemia type II (CDAll/HEMPAS): where are we now? Biochim Biophys Acta 2009, 1792:915-920

56. Paw BH, Davidson AJ, Zhou Y, Li R, Pratt SJ, Lee C, Trede NS, Brownlie A, Donovan A, Liao EC, Ziai JM, Drejer AH, Guo W, Kim CH, Gwynn B, Peters LL, Chernova MN, Alper SL, Zapata A, Wickramasinghe SN, Lee MJ, Lux SE, Fritz A Postlethwait JH, Zon LI: Cell-specific mitotic defect and dyserythropoiesis associated with erythroid band 3 deficiency. Nat Genet 2003, 34:59-64.

57. Iolascon A, Sabato V de Mattia D, Locatelli F: Bone marrow transplantation in a case of severe, type II congenital dyserythropoietic anaemia (CDA II). Bone Marrow Transplant 2001, 27:213-215.

58. Viel A, Branton D: Spectrin: on the path from structure to function. Curr Opin Cell Biol 1996, 8:49-55.

59. Liao EC, Paw BH, Peters LL, Zapata A, Pratt SJ, Do CP, Lieschke G, Zon LI: Hereditary spherocytosis in zebrafish riesling illustrates evolution of erythroid beta-spectrin structure, and function in red cell morphogenesis and membrane stability. Development 2000, 127:5123-5132

60. Cotter PD, Baumann M, Bishop DF: Enzymatic defect in 'X-linked' sideroblastic anemia: molecular evidence for erythroid deltaaminolevulinate synthase deficiency. Proc Natl Acad Sci U S A 1992 89:4028-4032.

61. Fukuda Y, Fujita $H$, Garbaczewski L, Sassa S: Regulation of $\beta$-globin mRNA accumulation by heme in DMSO-sensitive and DMSO-resistant murine erythroleukemia cells. Blood 1994, 83:1662-1667.

62. Igarashi K and Sun J: The heme-Bach1 pathway in the regulation of oxidative stress response and erythroid differentiation. Antioxid Redox Signal 2006, 8:107-118.

63. Brownlie A, Donovan A, Pratt SJ, Pratt SJ, Paw BH, Oates AC, Brugnara C, Witkowska HE, Sassa S, Zon LI: Positional cloning of the zebrafish sauternes gene: a model for congenital sideroblastic anaemia. Nat Genet 1998 20:244-250.

64. Franchini M: Hereditary iron overload: update on pathophysiology, diagnosis, and treatment. Am J Hematol 2006, 81:202-209.

65. Donovan A, Brownlie A, Zhou Y, Shepard J, Pratt SJ, Moynihan J, Paw BH, Drejer A, Barut B, Zapata A, Law TC, Brugnara C, Lux SE, Pinkus GS, Pinkus JL, Kingsley PD, Palis J, Fleming MD, Andrews NC, Zon LI: Positional cloning of zebrafish ferroportin 1 identifies a conserved vertebrate iron exporter Nature 2000, 403:776-781. 
66. Montosi G, Donovan A, Totaro A, Garuti C, Pignatti E, Cassanelli S, Trenor CC, Gasparini P, Andrews NC, Pietrangelo A: Autosomal-dominant hemochromatosis is associated with a mutation in the ferroportin (SLC11A3) gene. J Clin Invest 2001, 108:619-623.

67. Gordeuk VR, Caleffi A, Corradini E, Ferrara F, Jones RA, Castro O, Onyekwere O, Kittles R, Pignatti E, Montosi G, Garuti C, Gangaidzo IT, Gomo ZA, Moyo VM, Rouault TA, MacPhail P, Pietrangelo A: Iron overload in Africans and AfricanAmericans and a common mutation in the SCL40A1 (ferroportin 1) gene. Blood Cells Mol Dis 2003, 31:299-304.
68. Weiss LP, Wislocki GB: Seasonal variations in hematopoiesis in the dermal bones of the nine-banded armadillo. Anat Rec 1956, 126:143-163.

doi:10.1186/scrt146

Cite this article as: Kulkeaw K, Sugiyama D: Zebrafish erythropoiesis and the utility of fish as models of anemia. Stem Cell Research \& Therapy 2012, 3:55. 